\title{
Photoproduction of Multijets and Jets with Rapidity Gaps at HERA
}

\author{
Alexander A. Savin * \\ University of Wisconsin-Madison \\ 1150 University Ave., Madison, WI 5370-1390, USA
}

\begin{abstract}
Three- and four-jet final states have been measured in photoproduction at HERA. Cross sections have been studied as functions of different kinematic variables and compared to predictions of leading-order models with and without mupli-parton interactions. Photoproduction of dijet events with large rapidity gap between jets shows a clear excess over the predictions of standard MC models. MC models which include a strongly interacting exchange of a color-single object are able to describe the data.
\end{abstract}

\section{Three- and four-jet photoproduction}

In photoproduction (PHP) at HERA, a quasi-real photon, which is emmitted by the incoming positron, interacts with a parton from the proton.

In leading order(LO) approach the photon can interact as a point-like particle, so called direct PHP, or can fluctuate into a partonic system, and subsequently transfer only a fraction of its momentum in the hard interaction, so called resolved PHP.

The hadron-like structure of the photon in resolved events gives rise to the possibility of multi-hadron interactions (MPI), where more then one pair of partons from the incoming hadrons may interact with each other.

Figure 1 presents four-jet-production cross section as a function of $x_{\gamma}^{\text {obs }}$, where $x_{\gamma}^{o b s}$ stays for the fraction of the photon's momentum that is exchanged in the interaction. Jets were found in the pseudorapidity region $\left|\eta^{j e t}\right| \leq 2.4$ in the laboratory frame with $E_{T}^{\text {jet } 1,2} \geq 7$ and $E_{T}^{\text {jet } 3,4} \geq 5 \mathrm{GeV}$. The phase space was also restricted in elasticity to $0.2 \leq y \leq 0.85 . M_{n j}$ measures the invariant mass of the $n$-jet system.

Standard PHP MC predictions fail to

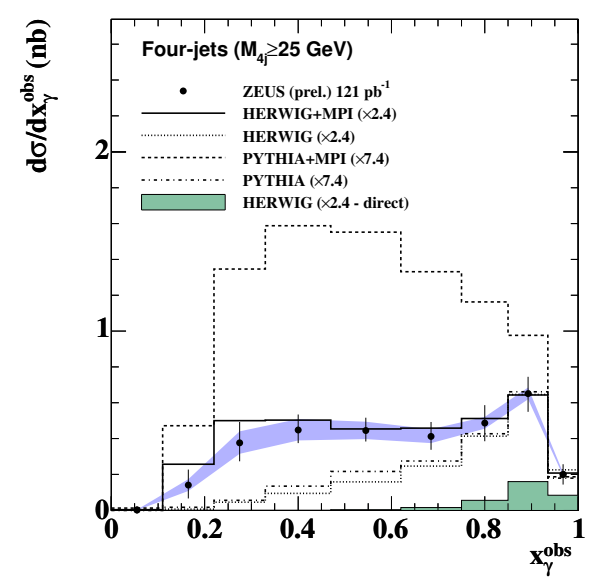

Figure 1: The four-jet-production cross section for $M_{4 j} \geq 25 \mathrm{GeV}$ differential in $x_{\gamma}^{\text {obs }}$. HeRWig and Pythia predictions with and without MPI, as well as HERWIG direct component, are shown. Shaded band represents the calorimeter energy scale uncertainty. describe the data. The MC underestimates the data in low- $x_{\gamma}^{\text {obs }}$, resolved enriched, region. Most pronounced difference is observed for the four-jet sample presented in Fig.1. The HERWIG model with MPIs, which was tuned to the three- and four-jet $x_{\gamma}^{o b s}$ and $M_{n j}$ data presented here, describes the data well. The

*On behalf of the ZEUS collaboration 
PythiA with MPIs tuned to generic collider data overestimates the cross sections. This problem was solved by retuning the model to the current data and new predictions will be shown in the final paper [2].

The cross sections differential in $M_{n j}$ presented in Fig. 2 demonstrate that the three-jet sample is well described by both MC samples with and without MPI. For the four-jet sample the standard MCs describe the high tail of the $M_{4 j}$, but significantly overestimate the cross section at low values of $M_{4 j}$. In contrast,
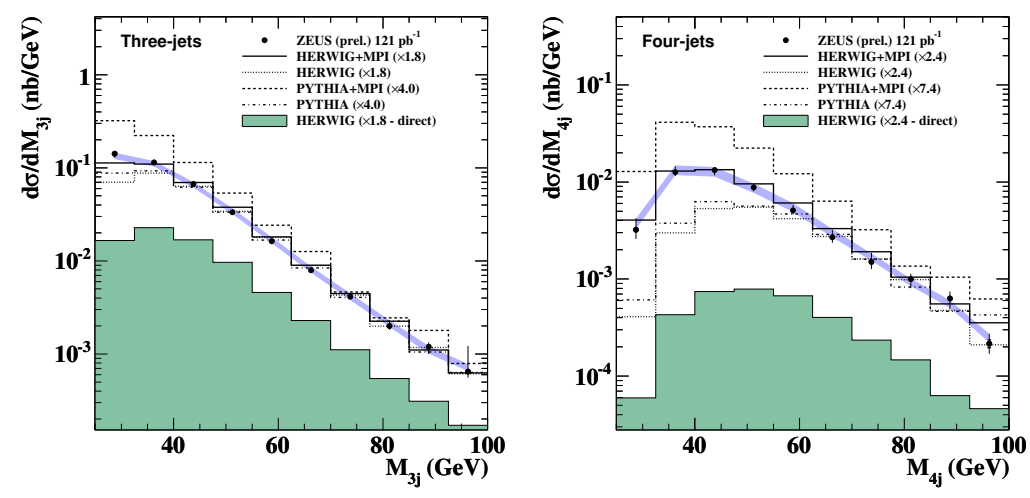

Figure 2: The three- and four-jet-production cross sections differential in $M_{n j}$. Other details as in Fig.1 the MC predictions with MPI give a reasonable good description of the data over the full $M_{4 j}$ range.

Figure 3 shows the $d \sigma / d y$ cross section for the three-jet sample. The shape of the distribution is governed by the available phase space. Both MC models without MPI describe the shape of the distribution reasonably well. Adding the MPIs have a significant effect causing the MC predictions to deviate from the data. One of the possible explanation may be the $y$-dependence of the MPI, which in this case will need to be revised.

The three-jet measurements were also compared with predictions of $O\left(\alpha \alpha_{S}^{2}\right)$ pQCD calculations by Klasen, Kleinwort and Kramer [3]. The calculation is LO for this process. Both hadronisation and MPI corrections obtained using the average corrections taken from the two MC models were applied to the calculation. The theo-

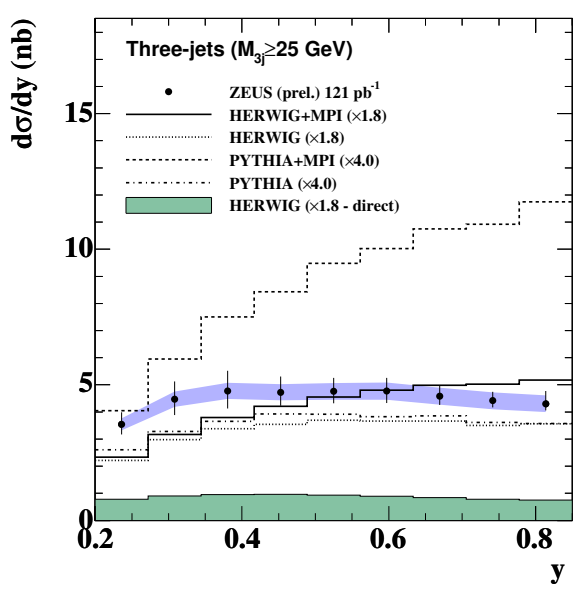
retical uncertainties were found to be large. The overall magnitude and shape of the calculation largely agree with the data within large uncertainties. The description of the data was found to be much worse if the predictions were not corrected for the effects of MPIs. 


\section{Events with rapidity gaps between jets}

The dominant mechanism for the production of jets with high transverse energy in hadronic collisions is a hard interaction between partons in the incoming hadrons via a quark or gluon propagator.

The exchange of color quantum numbers generally gives rise to jets in the final state that are color connected to each other and to the remnants of the incoming hadrons. This leads to energy flow populating the pseudorapidity region both between the jets and the hadronic remnants, and between the jets themselves. The fraction of events with little or no hadronic activity between the jets, gap fraction, is expected to be exponentially suppressed as the rapidity interval between the jets, $\Delta \eta$, increases. A non-exponentially suppressed fraction of such events would therefore be a signature of the exchange of a color-singlet object.

Events with at least two jets with $E_{T}^{j e t 1}>6$ and $E_{T}^{j e t 2}>5 \mathrm{GeV}$ and other cuts described in [4] were selected for this analysis. The transverse energy in the gap, $E_{T}^{G A P}$, was calculated by summing up the transverse energy of all jets, without any cut on $E_{\mathrm{T}}^{\mathrm{jet}}$, lying in the pseudorapidity region between the two highest- $E_{\mathrm{T}}^{\text {jet }}$ jets.

The inclusive dijet cross section as a function of $E_{\mathrm{T}}^{\mathrm{GAP}}$ is presented in Fig. 4 . At low $E_{\mathrm{T}}^{\mathrm{GAP}}$ values, where the colorsinglet (CS) contribution should be most pronounced, the data demonstrate a clear excess over the non-CS (NCS) MC predictions towards small $E_{\mathrm{T}}^{\mathrm{GAP}}$ values. In order to estimate the amount of color-singlet contribution, the direct and resolved components of each $\mathrm{MC}$ were mixed according to their predicted $\mathrm{MC}$ cross sections to give the NCS MC sample. The NCS and CS MC samples were then fitted to the data according to

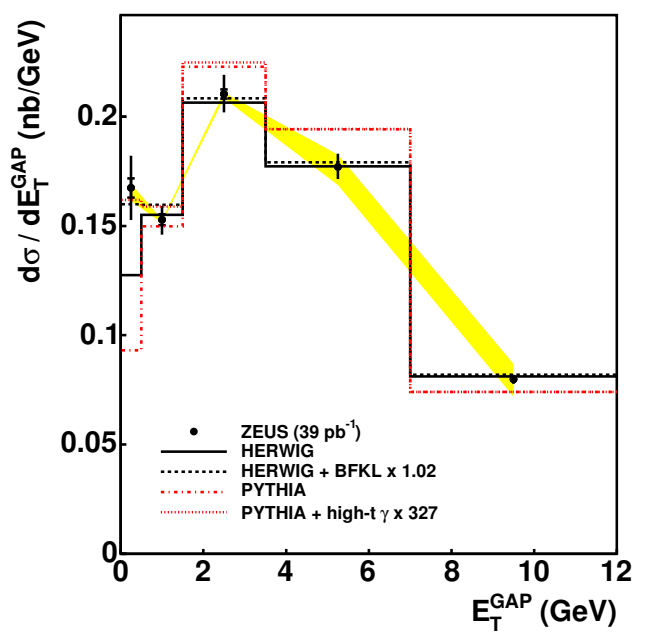

Figure 4: The inclusive dijet cross section, differential in $E_{\mathrm{T}}^{\mathrm{GAP}}$. The lines show predictions of HERwig and PYTHIA with and without CS exchange. The band shows the calorimeter energy-scale uncertainty.

$$
\frac{d \sigma}{d E_{\mathrm{T}}^{\mathrm{GAP}}}=P_{1} \frac{d \sigma^{\mathrm{NCS}}}{d E_{\mathrm{T}}^{\mathrm{GAP}}}+P_{2} \frac{d \sigma^{\mathrm{CS}}}{d E_{\mathrm{T}}^{\mathrm{GAP}}},
$$

where $P_{1}$ and $P_{2}$ were the free parameters of the fit. The best fit to the data resulted in $P_{1}=1.31 \pm 0.01$ and $P_{2}=327 \pm 20$ for PYTHIA and $P_{1}=1.93 \pm 0.01$ and $P_{2}=1.02 \pm 0.13$ for HERwiG.

The large value of $P_{2}$ for PYTHIA reflects the very low cross section of the high- $t$ photon exchange, which is not expected to represent the mechanism of strongly-interacting CS exchange and was only used to compare the data to an alternative CS model. In HERWIG the CS exchange was implemented using the LLA BFKL model by Mueller and Tang [5].

The color-singlet contribution to the total cross section, estimated by integrating the MC predictions over the entire $E_{\mathrm{T}}^{\mathrm{GAP}}$ range, was $(2.75 \pm 0.10) \%$ for PYTHIA and $(2.04 \pm 0.25) \%$ for HERWIG, where the errors represent only the statistical uncertainties of the fit. 
Figure 5 shows the gap fraction as a function of $\Delta \eta$ for the two regions of $E_{T}^{\mathrm{GAP}}$. For $E_{T}^{\mathrm{GAP}}<0.5 \mathrm{GeV}$ the data are consistent with a flat distribution in $\Delta \eta$. For higher $E_{T}^{\mathrm{GAP}}$ values the data first fall and then level out as $\Delta \eta$ increases The predictions of Pythia and Herwig without color-singlet exchange lie below the data over the entire $\Delta \eta$ range. With the addition of the color-singlet contribution, both MC models describe the data well.

For comparison with other experiments and $p \bar{p}$ measurements, which are expected to be similar to the resolved-photon process, the cross sections and gap fraction were also measured as function of $x_{\gamma}^{o b s}$ (not shown). The gap fraction decreases with decreasing $x_{\gamma}^{o b s}$ and the data are reasonably described by both MC models only after including the CS contribution, especially in the resolved photon region, $x_{\gamma}^{\text {obs }}<$ 0.75 , and at low $E_{T}^{\mathrm{GAP}}$.
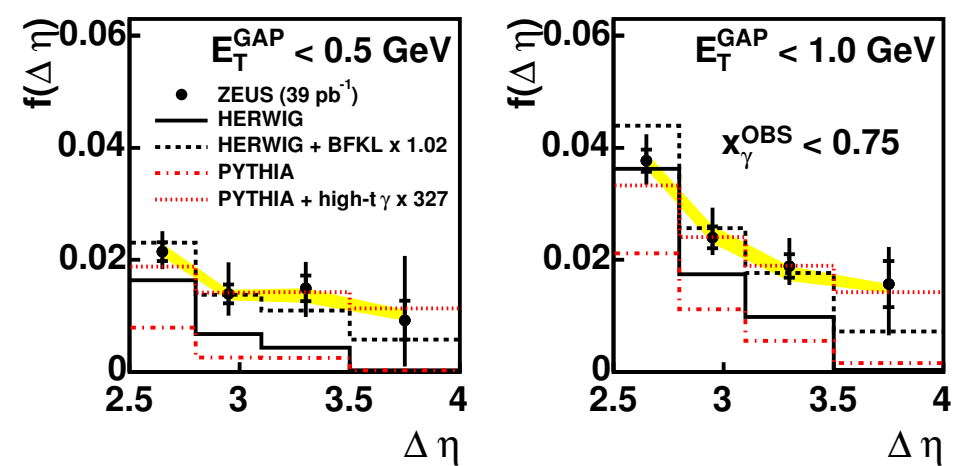

Figure 6: The gap fraction, $f$, as a function of $\Delta \eta$ in the resolved enriched region.

Figure 6 shows the gap fractions as a function of $\Delta \eta$ for the resolved enriched sample. For $E_{T}^{\mathrm{GAP}}<0.5 \mathrm{GeV}$ and $E_{T}^{\mathrm{GAP}}<1.0 \mathrm{GeV}$, both $\mathrm{MC}$ models predict almost no contribution to the gap fractions from the non-color-singlet component at high values of $\Delta \eta$. Unfortunately large theoretical uncertainties and differences in the model predictions preclude a modelindependent determination of the color-singlet contribution from these distributions.

\section{References}

[1] Slides: http://indico. cern.ch/contributionDisplay $\cdot$ py? contribId=220\&sessionId=6\&conf Id=9499

[2] ZEUS Coll., paper in preparation.

[3] M. Klasen, T. Kleinwort and G. Kramer, Z. Phys. Rev.-e C1 (1998).

[4] ZEUS Coll., S. Chekanov et al., Eur. Phys. J. C50 283 (2007)

[5] A.H. Mueller and W.K. Tang, Phys.Lett. B310 123 (1992) 